
\title{
28 Research Square \\ Is the effect of Decitabine limited to hypomethylation and DNMTs?
}

\section{Sina Dalvand}

Shahid Sadoughi University of Medical Sciences and Health Services Yazd

\section{Amin Namdari}

Fasa University of Medical Science

Ashraf Alemi ( $\square$ alemi.ashraf@gmail.com )

Islamic Azad University of Aligodarz

\section{Mohammad Hassan Meshkibaf}

Fasa University of Medical Science

\section{Sam Setayesh}

Shahid Sadoughi University of Medical Sciences and Health Services Yazd

Mojtaba Haghi Karamallah

Shahid Sadoughi University of Medical Sciences and Health Services Yazd

Mohammad Farrokhifar

Kar Higher Education institute, Rafsanjan,Iran

\section{Research article}

Keywords: HDAC3 ; HDAC7 ; HL-60 ; NALM-6 ; Decitabine ; AZad

Posted Date: August 20th, 2019

DOI: https://doi.org/10.21203/rs.2.13184/v1

License: (c) (i) This work is licensed under a Creative Commons Attribution 4.0 International License.

Read Full License 


\section{Abstract}

Background: Histone modifications play a crucial role in chromatin structure. Among enzymes, which regulate these processes, histone deacetylases (HDACs) can remove acetyl groups from histone tails, thus increasing their interaction with DNA and leading to chromatin condensation. 5-Aza-2'-deoxycytidine (AZad) or Decitabine is a potent hypomethylating agent that incorporates into DNA and traps DNA methyltransferase in the form of a covalent protein-DNA adduct. Azad, not only change the gene expression through demethylation of the gene's promoter, but it also can change gene expression independently from DNA demethylation. So, the present study was to distinguish whether AZad in addition to inhibitory effects on DNA methyltransferase, can change HDAC3 and HDAC7 mRNA expression in NALM-6, HL-60 cancer cell lines. Methods: HL-60, NALM-6 and normal cells were cultured, and the treatment dose of the AZad was obtained $(1 \mu \mathrm{M})$ by the MTT test. Finally, HDAC3 and HDAC7 mRNA expression were measured by Real Time PCR in HL-60 and NALM- 6 cancerous cells before and after treatment. In addition, HDAC3 and HDAC7 mRNA expression in un-treated HL-60 and NALM-6 cancerous cells were compared to the normal cells. Results: Our result revealed that expression of HDAC3 and HDAC7, in HL-60 and NALM- 6 cells increases as compared to normal cells. After treatment of HL-60 and NALM- 6 cells with AZad, HDAC3 and HDAC7 mRNA expression were decreased significantly. Conclusions: Our data showed, the effects of AZad are not limited to direct hypomethylation of DNMTs but it can indirectly affect other epigenetic factors, such as HDACs activity, through converging pathways. Keywords: HDAC3 ; HDAC7 ; HL-60; NALM-6 ; Decitabine ; AZad

\section{Background}

Epigenetics changes is the reason why, all body cells have the same DNA but with the different gene expression pattern, which leads to the formation of different cells types (1). These changes are inherited and have no effect on DNA nucleotide sequence (2). There are multiple types of epigenetic mechanisms such as the regulation of non-coding RNA, DNA methylation; by addition of methyl groups to a part of the DNA molecule. Histone modifications; by addition of acetyl group, methyl group, phosphors groups, ADPribosylation, ubiquitylation, etc. $(3,4)$. Histones modifications can result in alteration of gene transcription, this modifications carry out by relevant enzymes (5). Generally, histones are proteins that contribute to pack DNA through the creation of nucleosomes. There are five recognized type of histones including $\mathrm{H} 1, \mathrm{H} 2 \mathrm{~A}, \mathrm{H} 2 \mathrm{~B}, \mathrm{H} 3, \mathrm{H} 4$ and $\mathrm{H} 5$. The histones $\mathrm{H} 2 \mathrm{~A}, \mathrm{H} 2 \mathrm{~B}, \mathrm{H} 3$ along with $\mathrm{H} 4$ form the nucleosomes. Histone $\mathrm{H} 5$ exist in specific area of the DNA and histone $\mathrm{H} 1$ is observed in the places between nucleosomes $(6,7)$. The principal enzymes for monitoring of histone regulation are Histone methyltransferases (HMTs), Histone demethylases (HDMs), Histone acetyltransferase (HATs) and Histone deacetylases (HDACs) (5). HDACs are classified into 4 groups; group I HDACs, including HDAC 1 , 2, 3 and 8, present in nucleus, group II HDACs, including HDAC 4, 5, 6, 7, 9 and 10, move between the nucleus and cytoplasm (8). Whereas, often class I HDACs are ubiquitously expressed, but the class lla HDACs are expressed in a limited number of various cells (9). HDACs do not connect to DNA directly, and it is believed that to be recruited to special promoters via their interaction with DNA sequence-specific 
transcription agents (10). Some studies confirmed that HDAC inhibitors (HDACi) can cause apoptosis and stop cell cycle in acute lymphoblastic leukemia (ALL) cell cultures $(11,12)$. Some studies show that, HDAC7 and HDAC9 mRNA overexpression was recognized to be associated with bad prognostic agents and a higher risk of unfavorable results (13). Moreover, it has been shown that HDAC7 may control the beginning of apoptosis (14). High expression of HDAC7 also observed in pancreas adenocarcinomas (15). Thus, subsequently epigenetic drugs were developed to treat various diseases. There are many epigenetic drugs such as, bromodomain and inhibitors, HAT inhibitors, protein methyltransferase inhibitors, histone methylation inhibitors, methylation inhibiting and HDAC inhibitors drugs. 5-Aza-2'deoxycytidine (Azad) or Decitabine is an epigenetic drugs (16). In terms of molecular shape Azad is similar to deoxycytidine and usually used to demethylate of some gene's promoter, it is a powerful hypomethylating factor that combines into DNA and traps DNA methyltransferase in the form of a covalent protein-DNA adduct. And subsequently result in activated silent tumor-suppressor genes, growth-inhibitory, DNA repair and finally induce apoptosis $(17,18)$. Various scientific studies show that Azad, not only change the gene expression through demethylation of the gene's promoter, but it also can change gene expression independently from DNA demethylation. The effect of Azad on same gene expression in various environmental conditions can be different, and it can be the reason why patients treated with Azad show a variety responses (19). Different studies showed unusual DNA methylation and histone deacetylation is associated with tumorigenesis. Hence DNA methyltransferases (DNMTs) and histone deacetylases were identified as antitumor agents (20). Therefore, the purpose of our study was to distinguish whether Azad in addition to its inhibitory effects on DNA methyltransferases, can reduce HDAC3 and HDAC7 mRNA expression in NALM-6 (B cell precursor leukemia cell line), HL-60 (human promyelocytic leukemia cell line) cancer cell lines.

\section{Methods}

\section{Chemicals:}

The chemical such as Decitabine, RPMI 1640, Fetal bovine serum (FBS), Penicillin and streptomycin, Thiazolyl blue Tetrazolium Bromide (MTT), Eva green, DNA safe stain, RNA extraction kit, cDNA Synthesis Kits were purchase from Sigma Otsuka, America Pharmaceutical Inc., (Thermo scientific, MA, USA), (Gibco, Life technologies, Thermo fisher, USA), (Bio-idea, Iran), (Solis Biodyne, Estonia), (Biofact, Korea), (RNX-plus solution for total RNA isolation- sinaclon, Iran), (Thermo Fisher scientific, cDNA Inc. USA) respectively.

\section{Cell culture:}

Human cancer cell lines including NALM-6, HL-60 and normal cell (the Pasteur Institute, Tehran, Iran) were cultured in RPMI-1640 media enriched by $10 \%$ heat-inactivated fetal bovine serum (FBS), $50 \mathrm{U} / \mathrm{ml}$ penicillin and $50 \mu \mathrm{g} / \mathrm{ml}$ streptomycin at $37^{\circ} \mathrm{C}$ and $5 \% \mathrm{CO}_{2}$. We have obtained treatment does by the MTT assay and therefor NALM-6 and HL-60 cell lines were treated with $1 \mu$ M Decitabine for $24 \mathrm{~h}(21)$. 


\section{Cell viability assay (MTT test):}

Cells were cultured at a concentration of $1 \times 10^{5}$ cells/well in 96-well micro plate and treated with AZad at different concentrations $0.1 \mu \mathrm{M}, 1 \mu \mathrm{M}$ and $5 \mu \mathrm{M}$ for 24,48 and $72 \mathrm{~h}$ intervals. After treatment with AZad, $20 \mu \mathrm{l}$ of $5 \mathrm{mg} / \mathrm{ml}$ MTT solution was added to each well and incubated for $4 \mathrm{~h}$ at $37^{\circ} \mathrm{C}$. Next, $50 \mu \mathrm{l}$ of $20 \%$ acidified SDS added to the cells. Finally, absorbance of each well was measured at $570 \mathrm{~nm}$ by EPOCH Microplate Spectrophotometer (synergy HTX, BioTek, USA). Cell viability were expressed as a percentage in comparison to control. All tests were done 3 times $(21,22)$.

\section{Quantitative polymerase chain reaction (qPCR):}

Total RNA was isolated using an RNA extraction kit instruction protocol (RNX-plus solution for total RNA isolation, sinaclon, Iran). Similarly, cDNA was created using the transcriptor first strand cDNA synthesis kit (Thermo fisher scientific, cDNA Inc. USA). The expression level was calculated using the $2^{-\triangle \triangle C q}$ method (23). All experiments were repeated at least 3 times. The following primers for qPCR were used: HDAC3, forward 5'- CCA AGA CCG TGG CCT ATTT -3' and reverse 5'- AATGCAGGACCAGGCTATG -3'; HDAC7, forward 5'- GGACACCATGCAGATCATTCT -3' and reverse 5'- TGCACGTCC CAGTCTACAAT -3'; GAPDH, forward 5'- GAGCCACATCGCTCAGACAC -3' and reverse 5'- CATGTAGTTGAGGTCAATGAAGG -3'. Finally, all values were normalized to GAPDH expression levels.

\section{Statistical analysis:}

Statistical analysis was performed using two-tailed T-test. $P \leq 0.05$ was regarded as statistically significant. All statistical analyses were carried out by Graph Pad Prism 7.00 for windows (Graph Pad Software, San Diego, California, USA). Data were expressed as the mean \pm (SD) of at least three independent experiments.

\section{Results}

In the present study, we have investigated HDAC3 and HDAC7 gene expression in HL-60, NALM-6 and normal cells. We also check out the effect of AZad on HDAC3 and HDAC7 mRNA expression in HL-60 and NALM-6 cells after and before treatment. (Fig.1- 5). Our results show that HDAC3 (Fig. 1A) and HDAC7 (Fig. 1B) mRNA expression in $\mathrm{HL}-60$ cells increase significantly in comparison to normal cells. Whereas, HDAC3 (Figure 2A) and HDAC7 (Figure 2B) mRNA expression in HL-60 cells were declined after treatment with $1 \mu \mathrm{M}$ AZad. Similarly, HDAC3 (Figure 3A) and HDAC7 (Figure 3B) mRNA expression in NALM-6 cells elevated significantly when compared to normal cells. Nevertheless, HDAC3 (Figure 4A) and HDAC7 (Figure 4B) genes expression in NALM-6 cells was decreased after treatment with $1 \mu \mathrm{M}$ Azad. 


\section{Discussion}

Scientific studies show that low concentration of AZad allows the intracellular cycles to remain active (21). In contrast, cellular toxicity associated with higher concentrations of AZad is undesirable, due to secondary effect related to apoptosis induced by the drug that would interfere with the changes in primary gene expression. Low dose of AZad induces hypomethylation, and this effect disappears at high doses (24).

Epigenetic histone alterations play a significant role in chromatin structure. Among enzymes controlling these processes, histone deacetylases (HDACs) can eliminate acetyl groups from histone tails, thus enhancing their interaction with DNA and leading to chromatin condensation (25). According to a Kara Gianni P study (2007), enhanced expression of HDAC3 in Acute myeloid leukemia (AML) is correlated with poor prognosis and response to treatment (26), and also in the study by Moreno DA et al. (2010), enhanced expression of HDAC3 has also been in childhood acute lymphoblastic leukemia (ALL) (27). Overexpression of HDAC 3 in several cancer types has been observed to be correlated with poor prognosis and response to treatment, including: prostate, breast, ovarian and colorectal cancers as well as T-cell acute lymphoid leukemia (T-ALL) $(27,28)$. Several studies has shown that overexpression of HDAC7 and its interaction with MEF2 family genes in ALL mostly inhibits transcription in lymphoid cells, causing failure of normal differentiation in lymphoid lineage $(27,29)$. Overexpression of HDAC7 is correlated with a weak prognosis in chronic lymphoid leukemia (CLL). It binds to c-Myc transcription factor gene, causing its overexpression and enhanced proliferation of cancer cells in CLL (30). Our findings show that Decitabine significantly reduce HDAC3 and HDAC7 mRNA expression in HL-60 and NALM- 6 cancer cell lines. Specific HDAC inhibitors such as TSA (Trichostatin A) inhibits the Class I and II, but not Class III HDACs. TSA strongly inhibits HDAC1, HDAC2, HDAC3, HDAC4, HDAC6 and HDAC7 (13). While based on our results AZad reduce HDAC3 and HDAC7 mRNA expression. Therefore, it is suggested that the use of TSA and AZad at the same time may have synergistic effects and show better results, but it need more research. AZad is a powerful hypomethylating factor and Reducing HDAC3 and HDAC7 mRNA expression, we know the multiple therapy causes various side effects in patients. The use of multiple anticancer drugs is restricted due to toxicity in addition to the increase of drug resistance (31). Hence, new drugs are needed to ameliorate the clinical consequence and decrease the endurance to chemotherapy (32). Therefore, AZad can use as a candidate for hypomethylating and HDAC3 and HDAC7 reducing agents.

\section{Conclusions}

In summary, the results of the current study propose the possible involvement of HDAC3 and HDAC7 in leukemia's. Additional investigations are suggested to elucidate the potential role of HDAC3 and HDAC7 in carcinogenesis. These data showed that the effect of AZad are not limited to direct hypomethylation of DNMTs but also may indirectly affect other epigenetic factors, such as HDACs activity, through converging pathways. 


\section{Abbreviations}

- HDACs: histone deacetylases

- AZad:5-Aza-2'-deoxycytidine

- HMTs: Histone methyltransferases

- HDMs: Histone demethylases

- HATs: Histone acetyltransferase

- HDACs: Histone deacetylases

- HDACi : HDAC inhibitors

- ALL: acute lymphoblastic leukemia

- FBS: Fetal bovine serum

- MTT: Thiazolyl blue Tetrazolium Bromide

- qPCR: Quantitative polymerase chain reaction

- AML: Acute myeloid leukemia

- T-ALL: T-cell acute lymphoid leukemia

- CLL: chronic lymphoid leukemia

- TSA :Trichostatin A

\section{Declarations}

Ethics approval and consent to participate: This clinical protocol was approved by the Research Ethics Committee of the Shahid Sadoughi University of Medical Sciences (protocol number Ir.ssu.medicine.rec.1396.55). Signed informed consent was obtained from all individual participants included in the study. There are no human subjects or animals involved in the study.

Consent for publication: Not applicable.

Availability of data and materials: All data generated or analyzed during this study are included in this article.

Competing interests: the authors declare that they have no competing interests.

Funding: this study was financially supported by grant from the Shahid Sadoughi University of Medical Sciences, Yazd, Iran.

Authors' contributions: Sina Dalvand and Sam Setayesh conceived and designed the study. Amin Namdari performed the article search and data extraction. Mohammad Hassan Meshkibaf evaluated the methodological quality of each study. Mohammad Farrokhifar and Mojtaba Haghi Karamallah 
analyzed the data and wrote the paper, which was improved by Ashraf Alemi supervised the research. All authors read and approved the final manuscript.

Acknowledgment: Not applicable.

\section{Authors' Information:}

${ }^{1}$ International Campus, Department of Biochemistry and Molecular Biology, Shahid Sadoughi University of Medical Sciences, Yazd, Iran.

${ }^{2}$ Department of Biochemistry and Molecular Biology, School of Medicine, Shahid Sadoughi University of Medical Sciences, Yazd, Iran.

${ }^{3}$ Department of Clinical Biochemistry, Fasa University of Medical Sciences, Fasa, Fars, Iran

${ }^{4}$ Aligudarz School of Medical Sciences, Aligudarz Branch, Islamic Azad University, Aligudarz, Iran

${ }^{5}$ International Campus Biotechnology Research Center, Shahid Sadoughi University of Medical Sciences, Yazd, Iran.

${ }^{6}$ Kar Higher Education Institute, Rafsanjan, Iran

\section{References}

1. Sadikovic B A-RK, Squire J, Zielenska M. Cause and consequences of genetic and epigenetic alterations in human cancer. Current genomics. 2008;9(6):394-408.

2. Dupont C, Armant DR, Brenner CA, editors. Epigenetics: definition, mechanisms and clinical perspective. Seminars in reproductive medicine; 2009: () Thieme Medical Publishers.

3. Bhan A, Deb P, Mandal SS. Epigenetic code: histone modification, gene regulation, and chromatin dynamics. Gene regulation, epigenetics and hormone signaling London: Wiley. 2017.

4. Link A, Balaguer F, Goel A. Cancer chemoprevention by dietary polyphenols: promising role for epigenetics. Biochemical pharmacology. 2010;80(12):1771-92.

5. Baylin SB, Jones PA. A decade of exploring the cancer epigenome-biological and translational implications. Nature Reviews Cancer. 2011;11(10):726.

6. San EJ-E, Agirre X, Rodríguez-Otero P, Prosper F. Epigenetic regulation of cell signaling pathways in acute lymphoblastic leukemia. Epigenomics. 2013;5(5):525-38.

7. Burke MJ, Bhatla T. Epigenetic modifications in pediatric acute lymphoblastic leukemia. Frontiers in pediatrics. 2014;2:42.

8. Sharma S, Taliyan R. Histone deacetylase inhibitors: Future therapeutics for insulin resistance and type 2 diabetes. Pharmacological research. 2016;113:320-6. 
9. Zhou X, Marks PA, Rifkind RA, Richon VM. Cloning and characterization of a histone deacetylase, HDAC9. Proceedings of the National Academy of Sciences. 2001;98(19):10572-7.

10. Chinnadurai G. CtBP, an unconventional transcriptional corepressor in development and oncogenesis. Molecular cell. 2002;9(2):213-24.

11. Romanski A, Bacic B, Bug G, Pfeifer H, Gul H, Remiszewski S, et al. Use of a novel histone deacetylase inhibitor to induce apoptosis in cell lines of acute lymphoblastic leukemia. haematologica. 2004;89(4):419-26.

12. Sasaki K, Yamagata T, Mitani K. Histone deacetylase inhibitors trichostatin A and valproic acid circumvent apoptosis in human leukemic cells expressing the RUNX1 chimera. Cancer science. 2008;99(2):414-22.

13. Witt O, Deubzer HE, Milde T, Oehme I. HDAC family: What are the cancer relevant targets? Cancer letters. 2009;277(1):8-21.

14. Bakin RE, Jung MO. Cytoplasmic sequestration of HDAC7 from mitochondrial and nuclear compartments upon initiation of apoptosis. Journal of Biological Chemistry. 2004;279(49):51218-25.

15. Ouaïssi M, Sielezneff I, Silvestre R, Sastre B, Bernard J-P, Lafontaine JS, et al. High histone deacetylase 7 (HDAC7) expression is significantly associated with adenocarcinomas of the pancreas. Annals of Surgical Oncology. 2008;15(8):2318-28.

16. Heerboth S, Lapinska K, Snyder N, Leary M, Rollinson S, Sarkar S. Use of epigenetic drugs in disease: an overview. Genetics \& epigenetics. 2014;6:GEG. S12270.

17. Toyota M, Kopecky KJ, Toyota M-O, Jair K-W, Willman CL, Issa J-PJ. Methylation profiling in acute myeloid leukemia. Blood. 2001;97(9):2823-9.

18. Plumb JA, Strathdee G, Sludden J, Kaye SB, Brown R. Reversal of drug resistance in human tumor xenografts by 2'-deoxy-5-azacytidine-induced demethylation of the hMLH1 gene promoter. Cancer research. 2000;60(21):6039-44.

19. Seelan RS, Mukhopadhyay P, Pisano MM, Greene RM. Effects of 5-Aza-2'-deoxycytidine (decitabine) on gene expression. Drug metabolism reviews. 2018;50(2):193-207.

20. Conway SJ, Woster PM, Greenlee WJ, Georg G, Wang S. Epigenetics: novel therapeutics targeting epigenetics. ACS Publications; 2016.

21. Alemi A, Farrokhifar M, Zare-Zardini H, Haghi Karamallah M. A Comparison between the Anticancer Activities of Free Paclitaxel and Paclitaxel-Loaded Niosome Nanoparticles on Human Acute Lymphoblastic Leukemia Cell Line Nalm-6. Iranian journal of Pediatric Hematology and Oncology. 2018;8(3):153-60.

22. Alemi A, Zavar Reza J, Haghiralsadat F, Zarei Jaliani H, Haghi Karamallah M, Hosseini SA, et al. Paclitaxel and curcumin coadministration in novel cationic PEGylated niosomal formulations exhibit enhanced synergistic antitumor efficacy. Journal of nanobiotechnology. 2018;16(1):28.

23. Bustin SA, Benes V, Garson JA, Hellemans J, Huggett J, Kubista M, et al. The MIQE guidelines: minimum information for publication of quantitative real-time PCR experiments. Clinical chemistry. 2009;55(4):611-22. 
24. Momparler RL, Samson J, Momparler LF, Rivard GE. Cell cycle effects and cellular pharmacology of 5-aza-2'-deoxycytidine. Cancer chemotherapy and pharmacology. 1984;13(3):191-4.

25. Forsberg EC, Bresnick EH. Histone acetylation beyond promoters: long-range acetylation patterns in the chromatin world. Bioessays. 2001;23(9):820-30.

26. Karagianni P, Wong J. HDAC3: taking the SMRT-N-CoRrect road to repression. Oncogene. 2007;26(37):5439.

27. Moreno DA, Scrideli CA, Cortez MAA, De Paula Queiroz R, Valera ET, Da Silva Silveira V, et al. Differential expression of HDAC3, HDAC7 and HDAC9 is associated with prognosis and survival in childhood acute lymphoblastic leukaemia. British journal of haematology. 2010;150(6):665-73.

28. Weichert W. HDAC expression and clinical prognosis in human malignancies. Cancer letters. 2009;280(2):168-76.

29. Satyaraj E, Storb U. Mef2 proteins, required for muscle differentiation, bind an essential site in the Ig $\lambda$ Enhancer. The Journal of Immunology. 1998;161(9):4795-802.

30. Van Damme M, Crompot E, Meuleman N, Mineur P, Bron D, Lagneaux L, et al. HDAC isoenzyme expression is deregulated in chronic lymphocytic leukemia B-cells and has a complex prognostic significance. Epigenetics. 2012;7(12):1403-12.

31. Cheung-Ong K, Giaever G, Nislow C. DNA-damaging agents in cancer chemotherapy: serendipity and chemical biology. Chemistry \& biology. 2013;20(5):648-59.

32. Ma J, Guo X, Zhang S, Liu H, Lu J, Dong Z, et al. Trichostatin A, a histone deacetylase inhibitor, suppresses proliferation and promotes apoptosis of esophageal squamous cell lines. Molecular medicine reports. 2015;11(6):4525-31.

\section{Figures}




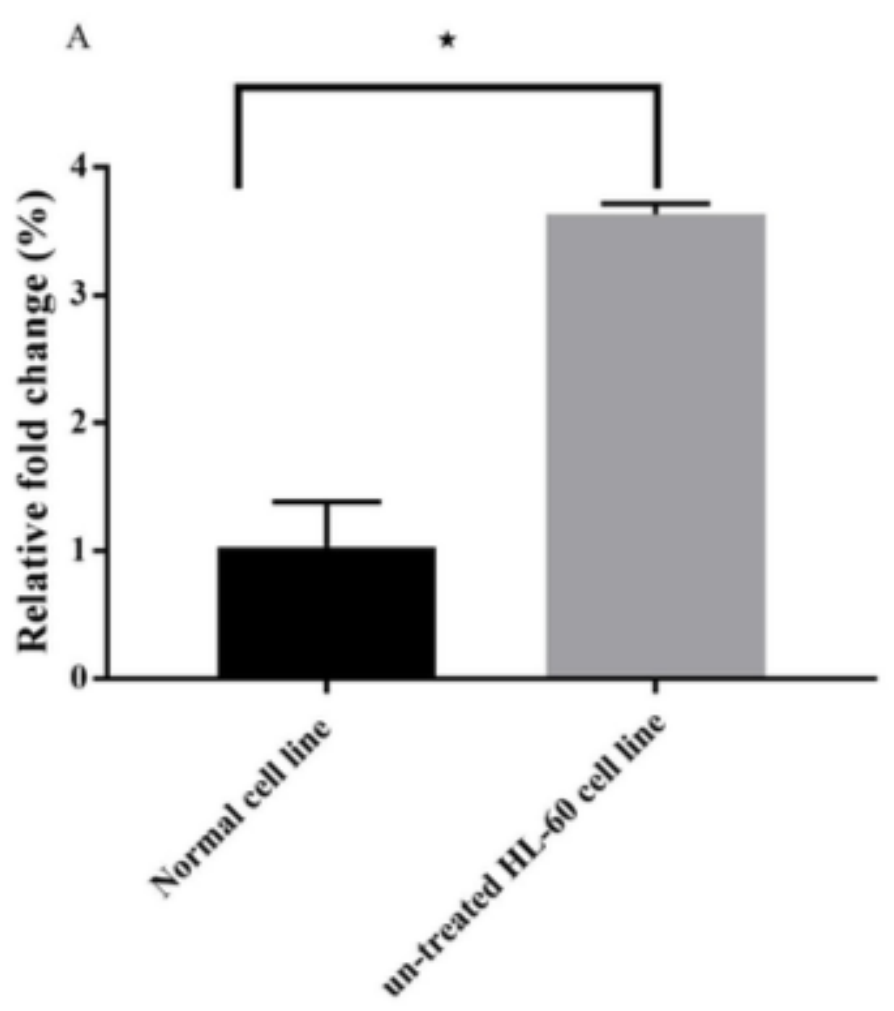

HDAC3 gene expression

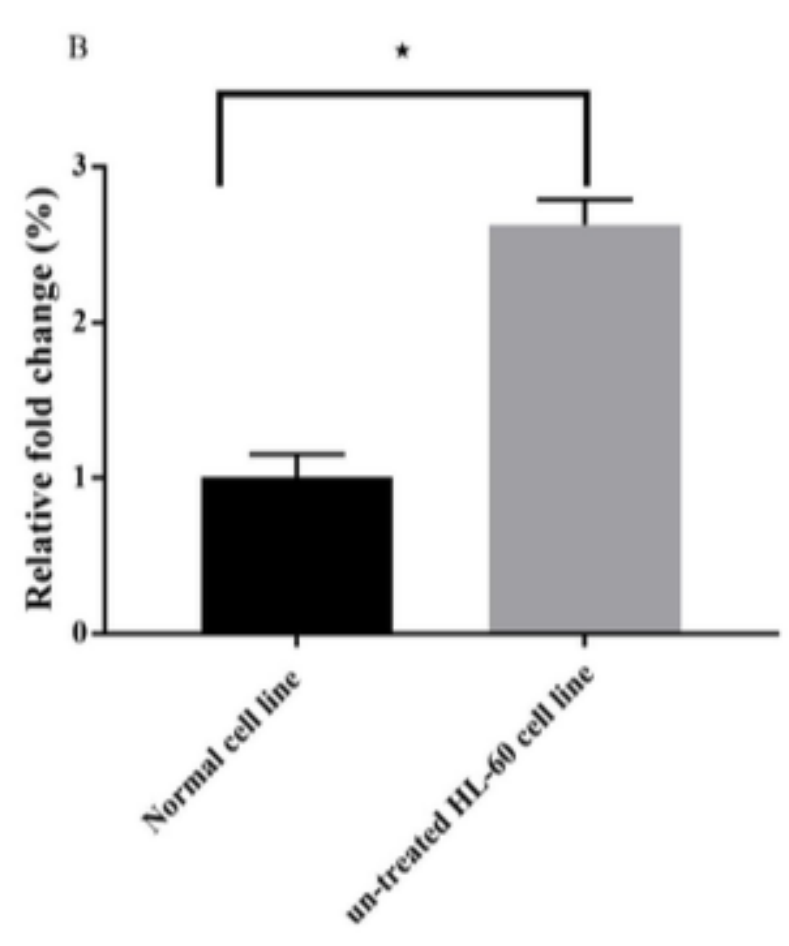

HDAC7 gene expression

\section{Figure 1}

The Relative fold changes of HDAC3 and HDAC7 gene expression in un-treated HL-60 cell line and in the healthy control group (as a calibrator), after normalization to GAPDH. A) The Relative fold changes of HDAC3 gene expression, in un-treated HL-60 cell line and in the healthy control group (as a calibrator), after normalization to GAPDH. B) Relative fold changes of HDAC7 gene expression, in un-treated HL-60 cell line and in the healthy control group (as a calibrator), after normalization to GAPDH. Data were considered significantly with $p<0.05$. ${ }^{*} P<0.05$ vs. control cells. 
A

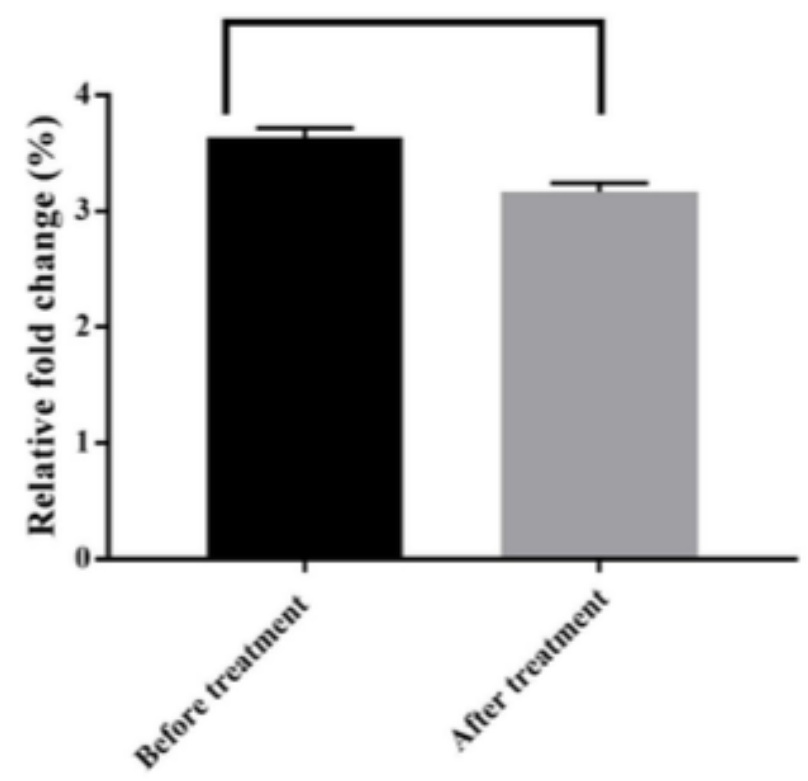

HDAC 3 gene expression in $\mathrm{HI}-60$ cell line
B

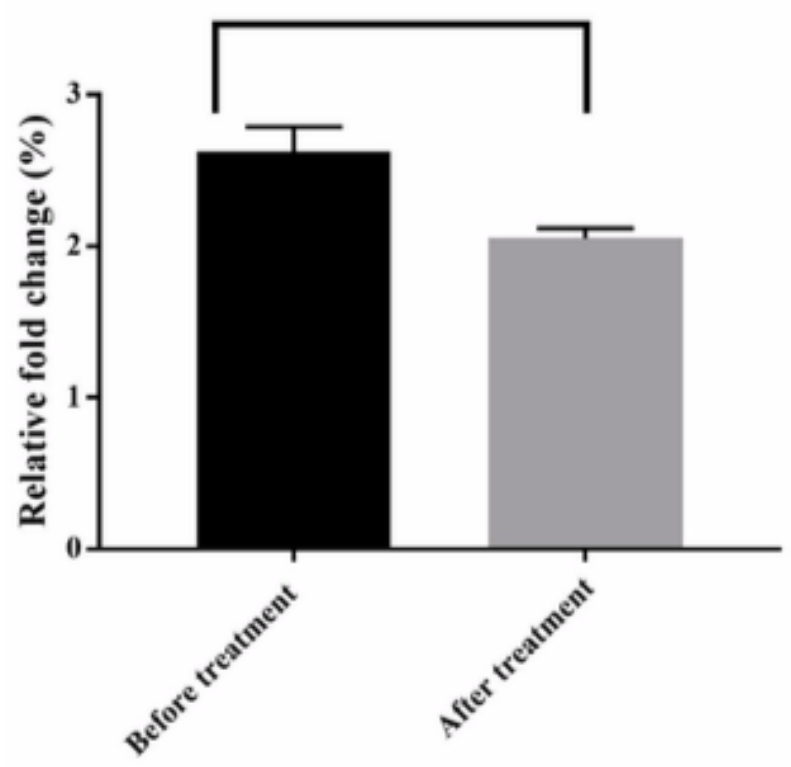

HDAC7 gene expression in $\mathrm{HI}-60$ cell line

\section{Figure 2}

The Relative fold changes of HDAC3 and HDAC7 gene expression in treated ( $1 \mu \mathrm{M} \mathrm{AZad)} \mathrm{HL-60} \mathrm{cell} \mathrm{line}$ and un-treated HL-60 cell line, after normalization to GAPDH. A) The Relative fold changes of HDAC3 gene expression, in treated ( $1 \mu \mathrm{M}$ AZad) $\mathrm{HL}-60$ cell line and un-treated HL-60 cell line, after normalization to GAPDH. B) Relative fold changes of HDAC7 gene expression, in treated HL-60 cell line and the treated $(1 \mu \mathrm{M}$ AZad) HL-60 cell line, after normalization to GAPDH. Data were considered significantly with $p<0.05 .{ }^{*} P<0.05$ before treatment vs after treatment. 


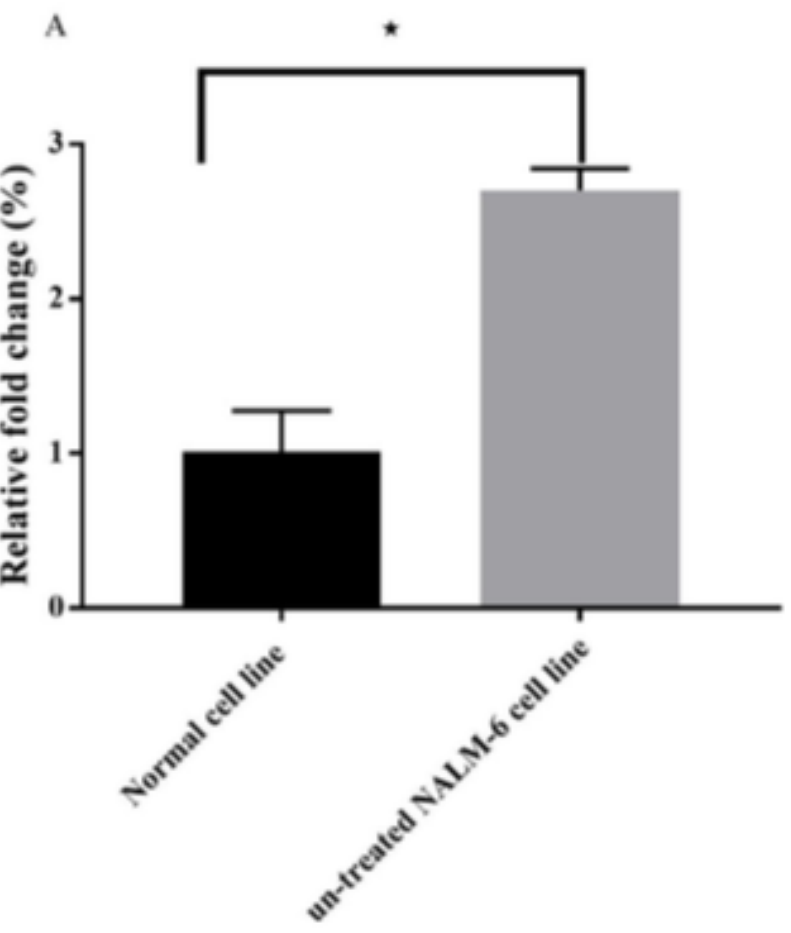

HDAC3 gene expression

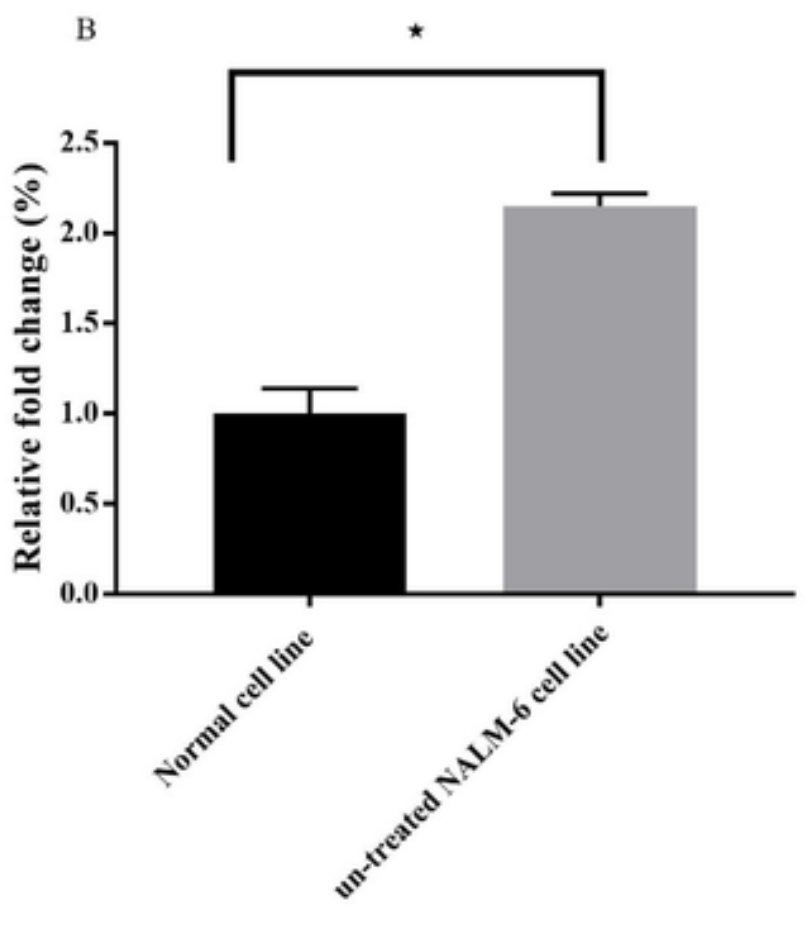

HDAC7 gene expression

Figure 3

The Relative fold changes of HDAC3 and HDAC7 gene expression in un-treated NALM-6 cell line and in the healthy control group (as a calibrator), after normalization to GAPDH. A) The relative fold changes of HDAC3 gene expression, in un-treated NALM- 6 cell line and in the healthy control group (as a calibrator), after normalization to GAPDH. B) The relative fold changes of HDAC7 gene expression, in un-treated NALM- 6 cell line and in the healthy control group (as a calibrator), after normalization to GAPDH. Data were considered significantly with $p<0.05 .{ }^{*}<<0.05$ vs. control cells. 


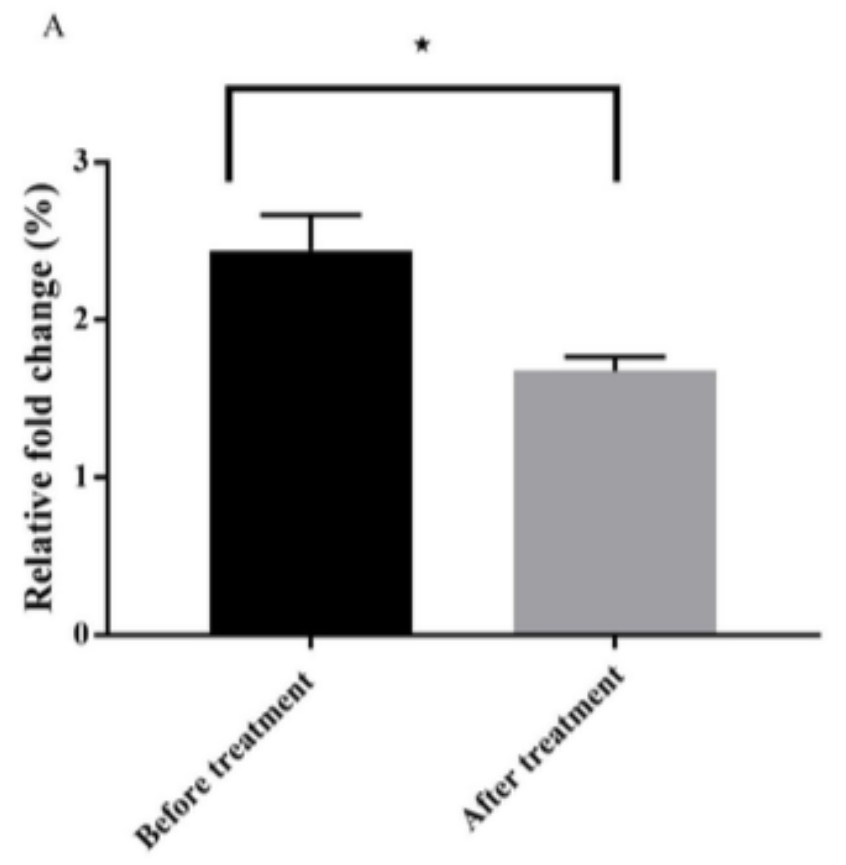

HDAC3 gene expression in NALM-6 cell line

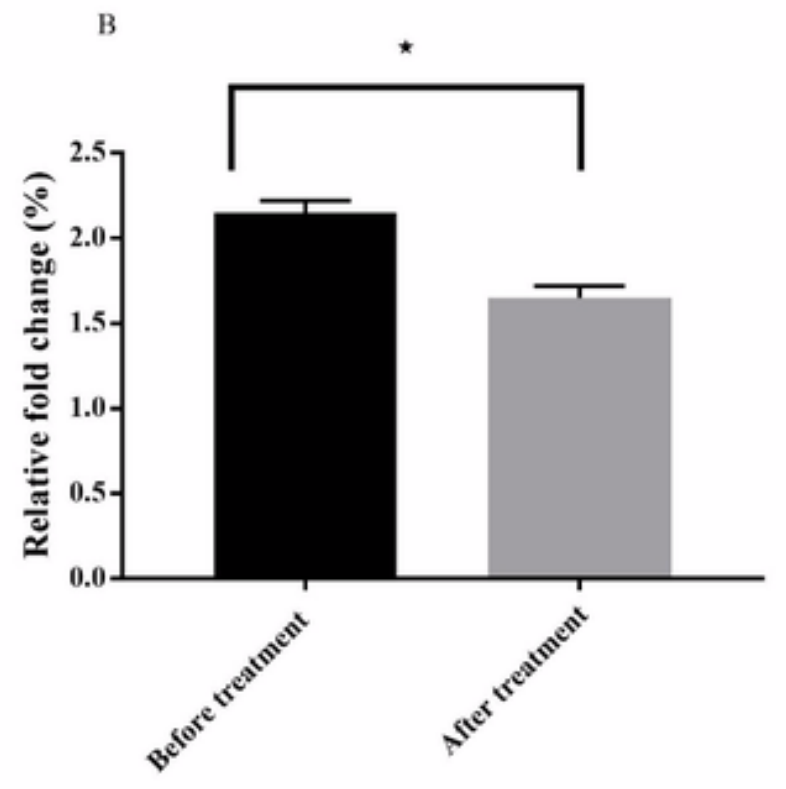

HDAC7 gene expression in NALM-6 cell line

\section{Figure 4}

The Relative fold changes of HDAC3 and HDAC7 gene expression in in treated ( $1 \mu \mathrm{M}$ AZad) NALM- 6 cell line and un-treated NALM- 6 cell line, after normalization to GAPDH. A) The relative fold changes of HDAC3 gene expression, in treated ( $1 \mu \mathrm{M}$ AZad) NALM- 6 cell line and the un-treated NALM- 6 cell line, after normalization to GAPDH. B) The relative fold changes of HDAC7 gene expression, in treated ( $1 \mu \mathrm{M} \mathrm{AZad)}$ NALM- 6 cell line and the un-treated NALM- 6 cell line, after normalization to GAPDH. Data were considered significantly with $p<0.05$. ${ }^{*}<0.05$ before treatment vs after treatment. 


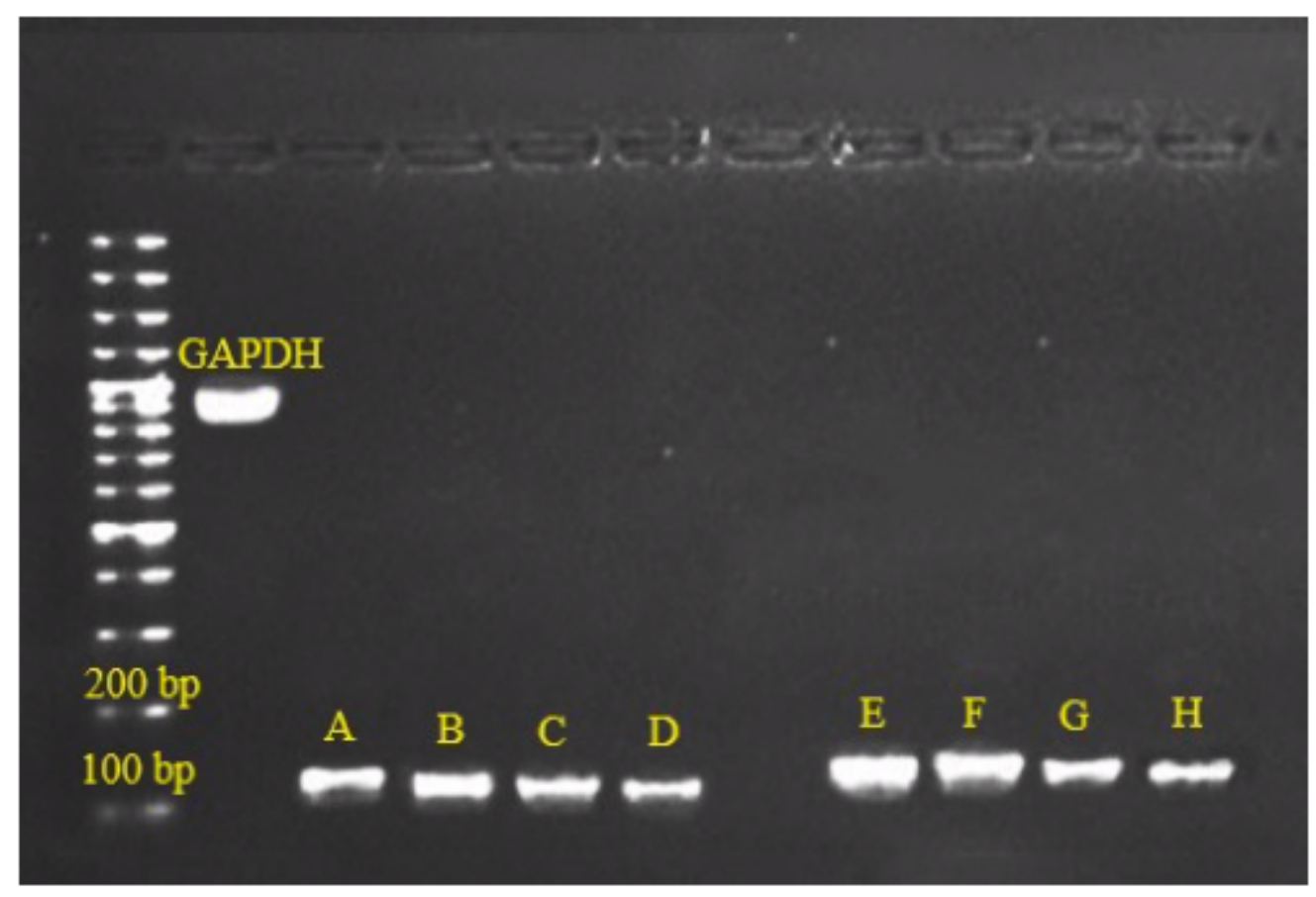

\section{Figure 5}

Real-Time PCR product. Represent Real-Time PCR gel patterns (qPCR) of HDAC3 and HDAC7, under nonsaturating conditions on a $3 \%$ Agarose gel and stained with ethidium bromide. Fig. 5A) HDAC3 genes expression in un-treat $\mathrm{HL}-60$ cell line (111 bp). Fig. 5B) HDAC3 genes expression in un-treat NALM-6 cell line (111 bp). Fig. 5C) HDAC3 genes expression in treated HL-60 cell line with $1 \mu \mathrm{M}$ AZad (111 bp). Fig. 5D) HDAC3 genes expression in treated NALM-6 cell line with $1 \mu \mathrm{M} \mathrm{AZad}(111 \mathrm{bp})$. Fig. 5E) HDAC7 genes expression in un-treat $\mathrm{HL}-60$ cell line (125 bp). Fig. 5F) HDAC7 genes expression in un-treat NALM- 6 cell line (125 bp). Fig. 5G) HDAC7 genes expression in treated HL-60 cell line with $1 \mu \mathrm{M}$ AZad (125 bp). Fig. $5 \mathrm{H}) \mathrm{HDAC7}$ genes expression in treated NALM-6cell line with $1 \mu \mathrm{M}$ AZad (125 bp). 